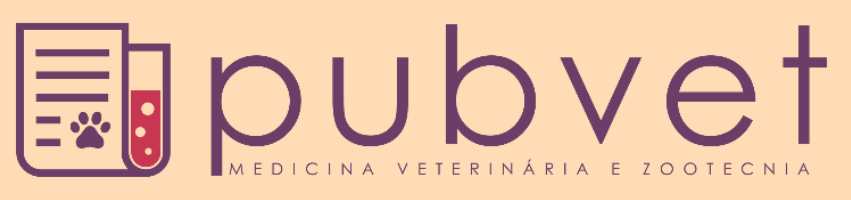

https://doi.org/10.31533/pubvet.v15n02a749.1-4

\title{
Microfilaruria por Dirofilária immitis em um cão na cidade de Uberlândia - Minas Gerais
}

\author{
Joao Helder Frederico de Faria Naves 91 , Poliana Ribeiro de Carvalho ${ }^{2} \oplus \mathbb{0}$, Thalita Fernandes \\ Fonseca $^{1}$, Mariana Rodrigues Moreira Guiotoku ${ }^{309}$ \\ ${ }^{I}$ Faculdade Presidente Antônio Carlos de Uberlândia, Departamento de Medicina Veterinária Preventiva, Uberlândia, Minas Gerais, Brasil. \\ ${ }^{2}$ Universidade Federal de Uberlândia, Departamento de Genética e Bioquímica, Uberlândia, Minas Gerais, Brasil. \\ ${ }^{3}$ Médica veterinária Autônoma, Uberlândia, Minas Gerais, Brasil. \\ *Autor para correspondência, E-mail: jhelder83@yahoo.com.br
}

Resumo. A Dirofilariose é considerada um importante problema de saúde pública, pois seu principal vetor são cães domésticos; existe uma grande proximidade desses cães com seus donos, a qual pode gerar uma possível transmissão cruzada. Diante disso é necessário que os profissionais da saúde estejam atentos e treinados a identificação clínica e laboratorial, a fim de não a negligenciar. O objetivo é descrever o caso de Dirofilaria immitis em cão na cidade de Uberlândia - MG, onde as larvas de microfilária foram encontradas inicialmente por um exame de urina na fase sedimentoscópica e microscopia sanguínea direta. Foram realizados os seguintes exames laboratoriais: hemograma completo, gota espessa, bioquímico e urinalise. Diversas alterações estavam presente no exame de urina, hematúria, piúria, hiperbilirrubinemia, proteinúria e presença de microfilária; outras alterações como: azotemia, parâmetros hematológicos que indicam anemia severa, leucocitose, neutrofilia e linfocitose. Existem poucos relatos de caso em que foi encontrado microfilárias no exame de urina de cães, assim, este relato auxilia na tomada de decisões e diagnóstico clínico mais preciso e aponta caminhos de investigação laboratorial, antes desconhecidas.

Palavras-chave: Dirofilaria immitis, sistema urinário, parasitose, zoonose

\section{Microfilaruria by Dirofilária immitis in a dog in the city of Uberlândia - Minas Gerais}

\begin{abstract}
Heartworm is considered an important public health problem, as its main vector is domestic dogs, there is great proximity of these dogs with their owners, which can generate a possible cross-transmission. Therefore, it is necessary that health professionals are attentive and trained in clinical and laboratory identification, in order not to neglect it. The objective is to describe the case of Dirofilaria immitis in a dog in the city of Uberlândia - MG, where the microfilaria larvae were initially found by a urine examination in the sedimentcopic phase and direct blood microscopy. Laboratory tests performed: complete blood count, thick drop, biochemical, urinalysis. Several changes were present in the urine test, hematuria, pyuria, hyperbilirubinemia, proteinuria, and the presence of microfilaria, other changes such as azotemia, hematological parameters that indicate severe anemia, leukocytosis, neutrophilia, and lymphocytosis. There are few case reports in which microfilariae were found in the examination of urine in dogs. This report assists in decision making and more accurate clinical diagnosis and points to previously unknown ways of laboratory investigation.
\end{abstract}

Keywords: Dirofilaria immitis, urinary system, larval cycle, parasitosis, zoonosis 


\title{
Microfilaruria por Dirofilária immitis en un perro en la ciudad de Uberlândia - Minas Gerais
}

\begin{abstract}
Resumen. El gusano del corazón se considera un problema importante de salud pública, ya que su vector principal son los perros domésticos, existe una gran proximidad de estos perros con sus dueños, lo que puede generar una posible transmisión cruzada. Por lo tanto, es necesario que los profesionales de la salud estén atentos y capacitados en identificación clínica y de laboratorio, para no descuidarlo. El objetivo es describir el caso de Dirofilaria immitis en un perro en la ciudad de Uberlândia - MG, donde las larvas de microfilaria se encontraron inicialmente mediante un examen de orina en la fase sedimentcópica y microscopía de sangre directa. Pruebas de laboratorio realizadas: examen de sangre completo, espesa gota de sangre, bioquímica, análisis de orina. Varios cambios estuvieron presentes en la prueba de orina, hematuria, piuria, hiperbilirrubinemia, proteinuria y la presencia de microfilaria, otros cambios como: azotemia, parámetros hematológicos que indican anemia severa, leucocitosis, neutrofilia y linfocitosis. Hay pocos informes de casos en los que se encontraron microfilarias en el examen de orina en perros. Este informe ayuda en la toma de decisiones y un diagnóstico clínico más preciso y apunta a formas de investigación de laboratorio previamente desconocidas.
\end{abstract}

Palabras clave: Dirofilaria immitis, sistema urinario, parasitosis, zoonosis

\section{Introdução}

A Dirofilaria immitis, parasita do sistema circulatório, tem sua disseminação facilitada em regiões que possuem climas tropicais e/ou temperados, como o Brasil (Santoro et al., 2019). O nematódeo realiza sua transmissão a partir de mosquitos hematófagos, este são provenientes da espécie Anophelles spp. da família Culicidae, composta por Culex pipiens, Culex quinquefasciatus, Aedes aegypti, Aedes albopictus, Anopheles maculipenis e Coquilettidia richiardi (Cirio, 2005; Costa da Silva \& Langoni, 2009).

A Dirofilariose é considerada uma doença emergente em consequência das diversas mudanças climáticas mundiais provenientes da interferência humana (Simón et al., 2012). Além de ser uma zoonose, ela também é um importante problema de saúde pública, pois seu principal vetor são cães domésticos; existe uma grande proximidade desses cães com seus donos, a qual pode gerar uma possível transmissão cruzada. Diante disso, é necessário que os profissionais da saúde, estejam atentos e treinados a identificação clínica e laboratorial, afim de não negligenciá-la (Al-Salihi et al., 2019).

O parasita que infecta os cães é a larva de microfilária que se desenvolve no intestino do mosquito, estas migram para as glândulas salivares do mosquito e por meio da sua picada, penetra na lesão provocada infectando o cão, já dentro de seu hospedeiro, tomando cerca de três meses para se tornarem larvas adultas e serem lançadas nas artérias pulmonares, após atingirem o coração levando cerca de seis à nove meses iniciam a produção de microfilárias e por meio do sistema circulatório são difundidas na corrente sanguínea (Bowman, 2010).

As microfilárias se fazem presente no sangue aproximadamente sete meses após a picada do mosquito infectado, podendo, nesta fase, ser realizado o teste de gota espessa de sangue, no qual pode ser identificado as microfilárias com o auxílio de um microscópio. O objetivo desse relato foi descrever o caso de Dirofilaria immitis em cão na cidade de Uberlândia - MG, onde as larvas de microfilária foram encontradas inicialmente por um exame de urina na fase sedimentoscópica e microscopia sanguínea direta.

\section{Materiais e métodos}

Foram coletadas amostras de urina e sangue de um cão de 6 anos, sem raça definida (SRD) e não castrado. Para detecção da larva de Dirofilaria immitis foi utilizada a técnica de gota espessa de sangue, a qual consiste em depositar gentilmente uma gota de sangue total em uma lâmina de vidro sobrepondo uma lamínula para ser realizada a leitura em microscópio. Também foi realizado a técnica de esfregaço sanguíneo corado com panótico, para identificação da microfilárias coradas. A leituras em microscópio 
foram realizadas em aumento de 40x. Além de avaliações hematológicas, foi realizado o exame de urinálise na qual a coleta foi realizada por cateterismo, exames bioquímicos como creatinina e ureia foram realizados, no equipamento semi automático do laboratório (Bioplus 200), e também foi realizado um hemograma (ABX micros 60).

\section{Resultados e discussão}

O animal apresentava sinais clínicos como apatia, desidratação, anúria, dor e sensibilidade a palpação de anormalidades na região inferior (próxima a bexiga e ânus) sendo constado hérnia perineal.

$\mathrm{Na}$ Tabela 1 consta alterações dos exames laboratoriais. No hemograma foi encontrado um quadro de anemia, com a queda nos parâmetros de hemácias, hemoglobina, volume glomerular (VG), microcitose, normocrômia. Foi detectado uma leucocitose com neutrofilia e linfocitose, no enquanto as plaquetas do animal se encontravam dentro do valor da normalidade.

Nos exames de urinálise, havia presença acentuada de leucócitos, hemácias, bilirrubina e proteínas, detectado presença de hematúria, e presença de larva de microfilária. Nos exames bioquímicos, o animal se encontrava em azotemia, sendo que os resultados de creatinina e ureia se encontravam elevados. As alterações dos parâmetros bioquímicos encontrados são comuns em cães que apresentam dirofilariose (Venco et al., 2005).

Tabela 1. Alterações de relevância nos exames laboratoriais solicitados do animal.

\begin{tabular}{ll}
\hline Exames realizados & Alterações \\
\hline Eritrograma & Microcítica e nomocrômica, hemácias, hemoglobina e VG baixas \\
Leucograma & Leucocitose com neutrofilia e linfocitose \\
Urinalise & Hematúria, piúria, hiperbilirrubinemia, proteinúria e presença de microfilária \\
Bioquímico & Creatinina e ureia aumentados \\
Gota espessa & Presença de microfilária \\
\hline
\end{tabular}

Após os exames, o diagnostico final apresentado pelo veterinário ao tutor foi de Dirofilariose, hérnia perineal (bexiga herniada) e doença renal crônica. Para continuidade do tratamento sugerido, foi de internação para realização de ecodopllercardiograma para identificar possíveis larvas de microfilária no coração e após o exame procedimento cirúrgico para correção de hérnia.

Em uma pesquisa utilizando 1023 cães, foram observados em 888 animais, baixos parâmetros hematológicos que indicam anemia, associados a neutrofilia (Niwetpathomwat et al., 2007), resultados que corroboram com os resultados vistos neste relato de caso. Em Uberlândia não existem muitos casos de dirofilariose relatados, e acredita-se que haja uma subnotificação pela falta de exames laboratoriais para caracterizar a doença.

A presença de hematúria e insuficiência renal aguda é frequentemente associadas, no entanto a presença de larvas na urina (microfilaruria) é extremamente rara, incomum e foram encontradas no animal em questão, fato esse que revela a evolução do microfilária no sistema excretor no animal, que dificulta ainda mais o tratamento e perspectiva de regressão do quadro do animal (Kaewthamasorn et al., 2008; Monobe et al., 2017).

Ocasionalmente, pode ocorrer bloqueios dos capilares renais por microfilárias, provocando glomerulonefrite (Urquhart et al., 1998), o que justificaria a presença da azotemia e aumenta o risco de falência renal no animal. Corroborando nossos achados bioquímicos na urina, os valores de creatinina e ureia também se apresentaram elevados em (Niwetpathomwat et al., 2007).

\section{Conclusão}

Este trabalho relatou a presença de microfilária de Dirofilária immitis na urina de um cão, o que geralmente é incomum para essa doença. Sendo a dirofilariose canina uma doença grave, de ocorrência mundial, um diagnóstico preciso e completo é de fundamental importância para dar ao animal um tratamento mais adequando, e mostrando ainda a importância de investigação de mais casos na nossa cidade, que tem poucos relatos da doença. 


\section{Referências}

Al-Salihi, K. A., Al-Dabhawi, A. H., Al-Rammahi, H. M., \& Kareem, F. A. (2019). Dirofilaria immitis infestation in imported police (K-9) dogs in Iraq: Brazilian Journal of Veterinary Research and Animal Science, 56(2), e152987. https://doi.org/10.11606/issn.1678-4456.bjvras.2019.152987

Cirio, S. M. (2005). Epidemiologia e clínica de cães portadores de dirofilariose em espaços urbanos de município do litoral do Paraná e aspectos da histologia de Culex quinquefasciatus Say, 1823 (Diptera, Culicidae) [Tese (Doutorado) - Programa de Pós-Graduação em Ciências Biológicas, Universidade Federal do Paraná]. http://hdl.handle.net/1884/27929

Bowman, D. Georgis (2010). Parasitologia Veterinária. Tradução de da $9^{a}$ Edição. 9. ed. Rio de Janeiro: Elsevier, 202-207.

Kaewthamasorn, M., Assarasakorn, S., \& Niwetpathomwat, A. (2008). Microfilaruria caused by canine dirofilariasis (Dirofilaria immitis): an unusual clinical presence. Comparative Clinical Pathology, 17(1), 61-65. https://doi.org/10.1007/s00580-007-0675-1

Costa da Silva, R. \& Langoni, H. (2009) Dirofilariose, zoonose emergente negligenciada. Ciência Rural, 39, (5). https://doi.org/10.1590/s0103-84782009005000062

Monobe, M. M., Silva, R. C., Araujo Junior, J. P., \& Takahira, R. K. (2017). Microfilaruria by Dirofilaria immitis in a dog: a rare clinical pathological finding. Journal of Parasitic Diseases, 41(3), 805-808. https://doi.org/10.1007/s12639-017-0892-8

Niwetpathomwat, A., Kaewthamasorn, M., Tiawsirisup, S., Techangamsuwan, S., \& Suvarnvibhaja, S. (2007). A retrospective study of the clinical hematology and the serum biochemistry tests made on canine dirofilariasis cases in an animal hospital population in Bangkok, Thailand. Research in Veterinary Science, 82(3), 364-369. https://doi.org/10.1016/j.rvsc.2006.09.002

Santoro, M., Miletti, G., Vangone, L., Spadari, L., Reccia, S., \& Fusco, G. (2019). Heartworm Disease (Dirofilaria immitis) in Two Roaming Dogs From the Urban Area of Castel Volturno, Southern Italy. Frontiers in Veterinary Science, 6, 270. https://doi.org/10.3389/fvets.2019.00270

Simón, F., Siles-Lucas, M., Morchón, R., González-Miguel, J., Mellado, I., Carretón, E., \& MontoyaAlonso, J. A. (2012). Human and Animal Dirofilariasis: the Emergence of a Zoonotic Mosaic. Clinical Microbiology Reviews, 25(3), 507 LP - 544. https://doi.org/10.1128/CMR.00012-12

Urquhart, G. M., Armour, J., Duncan, J. L. \& Jennings, F. W. (1998). Parasitologia veterinária. 2.ed. Rio de Janeiro: Guanabara Koogan.

Venco, L., Kramer, L., \& Genchi, C. (2005). Heartworm disease in dogs: Unusual clinical cases. Veterinary Parasitology, 133(2-3), 207-218. https://doi.org/10.1016/j.vetpar.2005.04.010

Histórico do artigo:

Aprovado: 8 de agosto de 2020.

Disponível online: 18 de dezembro de 2020
Licenciamento: Este artigo é publicado na modalidade Acesso Aberto sob a licença Creative Commons Atribuição 4.0 (CC-BY 4.0), a qual permite uso irrestrito, distribuição, reprodução em qualquer meio, desde que o autor e a fonte sejam devidamente creditados. 